\title{
Variações Na Estrutura Da Comunidade Zooplanctônica Próxima A Usina I Da Central Nuclear Almirante Álvaro Alberto (C.N.A.A.A.) - (Angra Dos Reis-R.J.-Brasil) - Ciclo 1980/81 E 1991/93.
}

\author{
Cristina de Oliveira Dias ${ }^{1 *}$; Sérgio L.C Bonecker. ${ }^{1}$ e Catarina R. Nogueira (in memoriam), \\ ${ }^{1}$ Departamento de Zoologia, Instituto de Biologia, U.F.R.J., C.C.S., Bloco A, Cidade Universitária, CEP: 21.941 - \\ 590, Rio de Janeiro, Brasil.
}

\begin{abstract}
During the years of 1980/80 was performed a ecological study in Ribeira Bay (Angra dos Reis-R.J.-Brazil) in order to provide informations about of the planktonic community in pre-operational conditions, in the region of the Admiral Álvaro Aberto Nuclear Power Plant (C.N.A.A.A.). After 10 years of activities of the Nuclear Power Plant the zooplankton material was obtained in vertical hauls with a net of $150 \mu \mathrm{m}$ at two fixed points (Piraquara de Fora Cove and Itaorna Beach) in order to study the zooplankton associations related to environmental variables and comparing with the anterior study. The zooplankton evolution showed a sazonal variation with the dominance of the holoplanktonic organisms. The Copepoda showed its the greatest growth during the autumn and the winter. During the summer and the spring its relative abundance suffered a progressive diminution when the Cladocera passed to be the more representative organisms without a competition between its. The sazonal variation presented by the organisms was related with the raining period that fall in the region.
\end{abstract}

Key words: Zooplâncton, Rio de Janeiro, Usina Térmica, Sazonal Variation.

\section{INTRODUÇÃO}

A baía da Ribeira está localizada no município de Angra dos Reis, litoral sul do Estado do Rio de Janeiro. Inserida na baía de Ilha Grande, apresenta características de zonas tropicais com verões chuvosos e invernos secos.

Durante o ano de 1980, a baía da Ribeira foi objeto de estudo detalhado com o levantamento taxonômico de sua fauna e flora marinhas (FUJB, 1981), visando-se a obtenção de dados ambientais, em condições pré-operacionais, na região da Usina I da Central Nuclear Almirante Álvaro Alberto (C.N.A.A.A.). A partir destes dados, Nogueira et al. (1987 e 1991) realizaram um levantamento preliminar das comunidades zooplanctônicas existentes, correlacionando-as com diversas variáveis ambientais.
A partir de 1986 foi iniciado um programa de monitoramento trimestral do zooplâncton em dois pontos fixos, na área de tomada de água usada para refrigeração do circuito secundário da Usina (Saco de Piraquara de Fora) e outro na região de lançamento do efluente da Usina (praia de Itaorna).

Esta estratégia de monitoramento vem sendo mantida com o objetivo de realizar um acompanhamento temporal da comunidade zooplanctônica e avaliar o impacto sobre a população, nas águas usadas na refrigeração dos condensadores da Usina.

Com o objetivo de avaliar a composição e variação da comunidade zooplanctônica, após 10 anos de funcionamento da Usina, foram estudados os dados coletados no ciclo 1991/93, comparando-os com aqueles levantados em 1980/81.

\footnotetext{
* Autor para correspondência
} 


\section{MATERIAL E MÉTODOS}

A região de estudo na baía da Ribeira, está localizada entre a Ponta do Pasto $\left(44^{\circ} 25^{\prime} 30^{\prime \prime} \mathrm{W}\right.$ e $\left.22^{\circ} 59^{\prime} 48^{\prime \prime} \mathrm{S}\right)$ e a Praia do Diego $\left(44^{\circ} 27^{\prime} 42^{\prime \prime} \mathrm{W}\right.$ e $\left.23^{\circ} 01^{\prime} 12^{\prime \prime S}\right)$. Foram realizadas amostragens em dois pontos fixos (Fig. 1), abrangendo dois ciclos de coleta: mensais no período que se estendeu de fevereiro/80 a janeiro/81 e trimestrais durante os anos de 1991 a 1993.

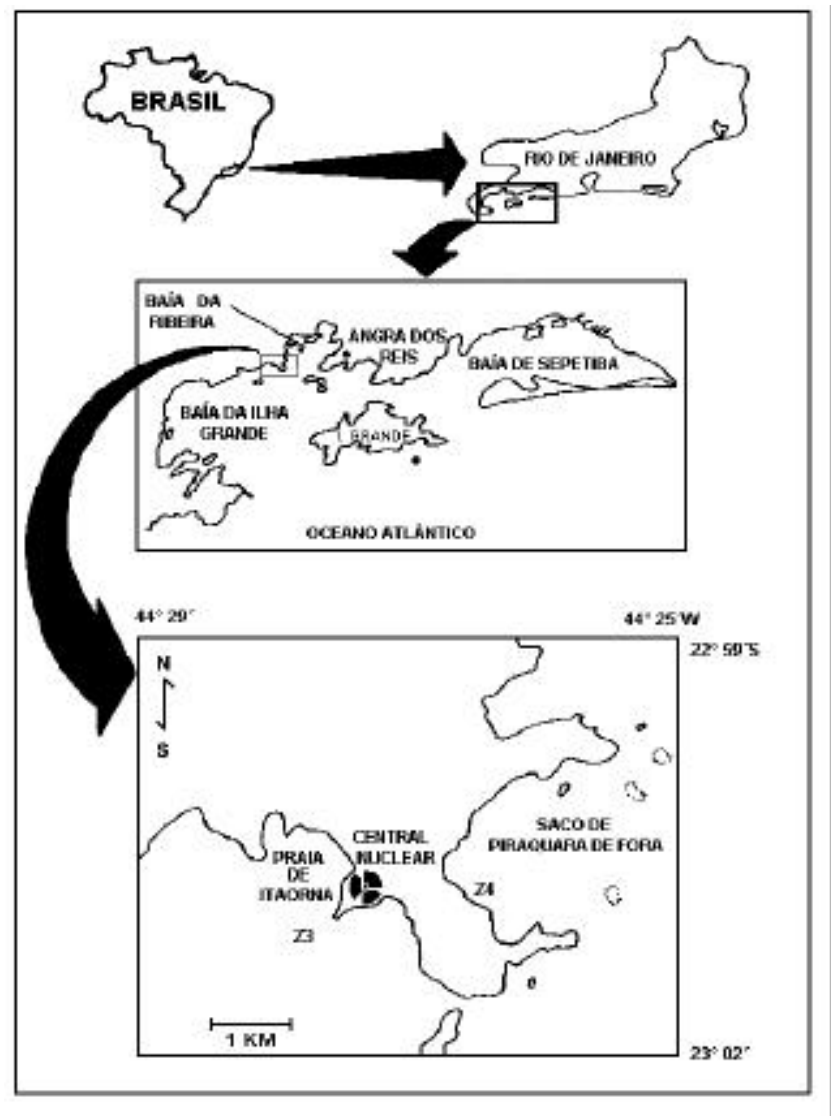

Figura 1: Mapa da área de estudo com a localização dos pontos de coleta (Z3 e Z4).

Em todas as campanhas foram realizadas medições de temperatura (termosalinômetro de Beckmann e termômetro de mercúrio), salinidade (termosalinômetro de Beckmann), oxigênio dissolvido (método de Winkler modificado) e clorofila a. Foram utilizados, também, os valores de precipitação total, fornecidos pela Assessoria de Segurança Ambiental (A.SA.N) de FURNAS Centrais Elétricas - Cadastro de Dados Meteorológicos (NCMAR23).
Para a coleta do plâncton foram realizados arrastos verticais ao longo dos $5 \mathrm{~m}$ da coluna d'água, com uma rede cônico-cilíndrica de 50 $\mathrm{cm}$ de diâmetro de boca, malha de $150 \mu \mathrm{m}$ e 150 $\mathrm{cm}$ de comprimento. As amostras foram fixadas, após a coleta, em solução de formaldeído diluído a $10 \%$ com água do mar, tamponado a razão de $20 \mathrm{~g} / \mathrm{l} \mathrm{com}$ tetraborato de sódio. O volume de água filtrada pela rede foi estimado à partir do produto do comprimento do cabo lançado $(5 \mathrm{~m})$ e a área da boca da rede $\left(0,20 \mathrm{~m}^{2}\right)$. No laboratório foram feitas triagem total dos organismos zooplanctônicos. Os grupos mais abundantes foram subamostrados. Foi determinada a composição taxonômica dos grupos zooplanctônicos mais abundantes (Copepoda, Cladocera, Appendicularia e Chaetognatha).

A análise quantitativa do material foi realizada utilizando a densidade dos organismos (ind. $\mathrm{m}^{-3}$ ). A diversidade específica foi calculada através do índice de Shannon-Weaver (LEGENDRE \& LEGENDRE, 1983).

Sobre a matriz de dados formada pela densidade das espécies zooplanctônicas identificadas (Copepoda, Cladocera e Chaetognatha), foi aplicada uma análise de grupamento ("Cluster Analysis"), utilizando-se o índice de BravaisPearson (LEGENDRE \& LEGENDRE, 1983).

\section{RESULTADOS}

\section{Primeiro ciclo de coleta $(\mathbf{1 9 8 0 / 8 1})$}

Os dados referentes à coleta, a variação anual e distribuição da população zooplanctônica durante o primeiro ciclo de coleta, assim como sua relação com as variáveis ambientais são encontrados em Nogueira et al. (1987 e 1991).

A baía da Ribeira apresentou temperaturas mínimas sempre superiores a $22^{\circ} \mathrm{C}$. As variações na salinidade na superfície, tiveram os valores compreendidos entre 35 e $36 \%$ de junho a setembro. As variações, no sentido vertical, da temperatura e da salinidade, foram pequenas não ultrapassando $1^{\circ} \mathrm{C}$ e $3 \%$, respectivamente. 
O estudo das variações anuais e sazonais do zooplâncton da baía da Ribeira, no período de fevereiro de 1980 a janeiro de 1981, evidenciou duas épocas de crescimento populacional: maio (outono) e novembro-dezembro (final de primavera e início de verão) (Figs. 2a e 2b).

O zooplâncton foi qualitativa $\mathrm{e}$ quantitativamente dominado pelos Cladoceros, de janeiro a maio e de novembro a dezembro, e pelos Copepoda, de junho a setembro.

Entre os Cladoceros, Penilia avirostris foi a espécie mais abundante, com até $75 \%$ de toda a população.

Os Copepoda constituíram de 50 a $90 \%$ do zooplâncton total. Um total de 21 espécies foram encontradas durante o estudo, sendo as mais importantes Paracalanus quasimodo, $\boldsymbol{P}$. indicus, $P$. crassirostris, Oithona hebes e Euterpina acutifrons, todas elas características de águas costeiras do litoral brasileiro (Tabela I).

Outros organismos presentes foram os Appendicularia, Chaetognatha, Hydromedusae, Siphonophorae e larvas de Crustacea.

Analisando a diversidade específica, calculada para as espécies de Copepoda, Cladocera e Chaetognatha, foi observado que o menor índice $\left(1,0\right.$ bits.ind $\left.^{-1}\right)$ foi registrado no outono (estação Z4), devido as altas densidades da espécie Penilia avirostris. Os maiores índices (acima de 3,0 bits.ind ${ }^{-1}$ ) foram registrados durante o inverno nas duas estações (Figs. 2a e2b).
A)

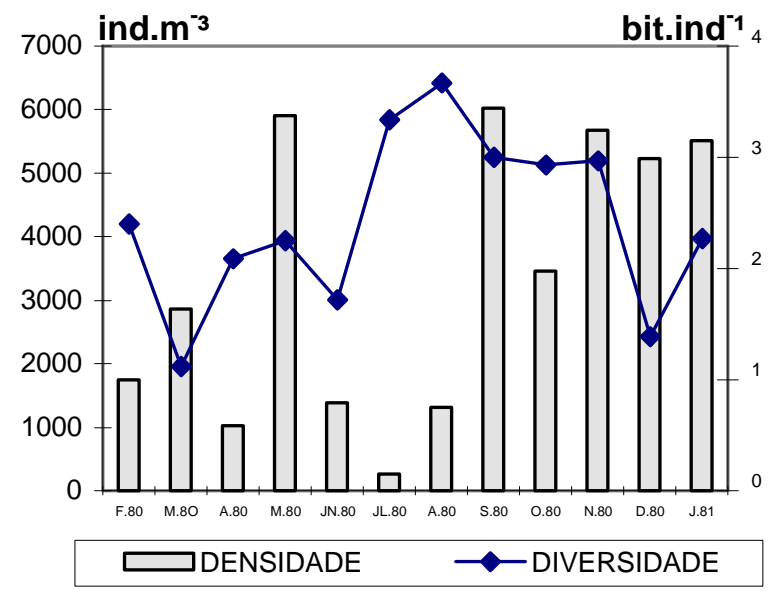

B)

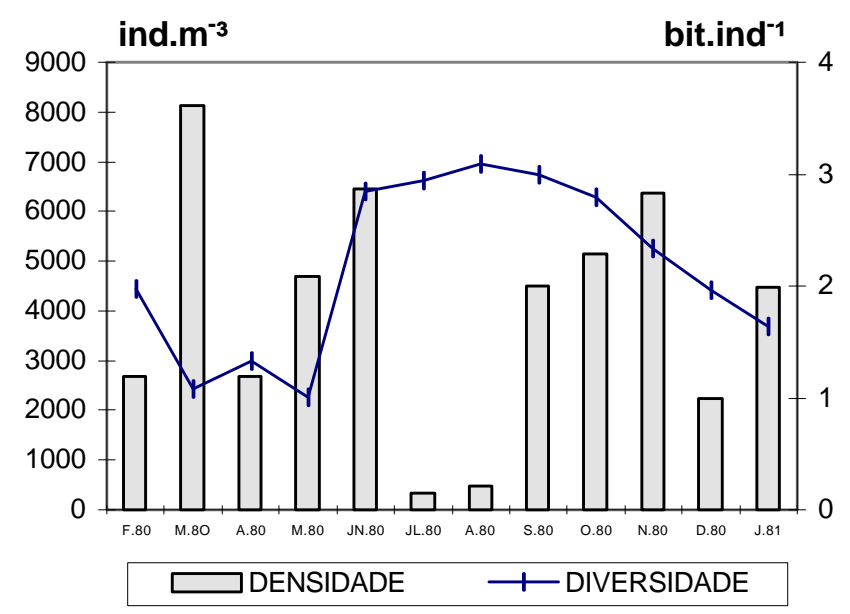

Figura 2: Variação dos valores de densidade e diversidade dos grupos zooplanctônicos ao longo do primeiro ciclo de coleta (1980/81): a) estação Z3 e b) estação Z4.

Tabela I: Ocorrência dos taxa zooplanctônicos nos dois ciclos de coleta (1980/81 e 1991/93).

\begin{tabular}{|c|c|c|c|c|c|c|c|c|c|}
\hline \multirow[b]{2}{*}{ TAXA } & \multicolumn{4}{|c|}{ COLETA } & \multirow[b]{2}{*}{ TAXA } & \multicolumn{4}{|c|}{ COLETA } \\
\hline & 80 & 91 & 92 & 93 & & 80 & 91 & 92 & 93 \\
\hline 1) Filo Annelida & & & & & Subordem Cladocera & & & & \\
\hline $\begin{array}{l}\text { Classe Polychaeta } \\
\text { 2) Filo Arthropoda }\end{array}$ & $X$ & $X$ & $X$ & $X$ & $\begin{array}{l}\text { Superfamília Sidoidea } \\
\text { Família Sididae }\end{array}$ & & & & \\
\hline $\begin{array}{l}\text { Subfilo Crustacea } \\
\text { Classe Branchiopoda }\end{array}$ & & & & & $\begin{array}{l}\text { Penilia avirostris } \\
\text { Superfamília Polyphemoidea }\end{array}$ & $X$ & $X$ & $x$ & $x$ \\
\hline
\end{tabular}

Ordem Diplostraca

cont. 


\begin{tabular}{|c|c|c|c|c|c|c|c|c|c|}
\hline \multirow[b]{2}{*}{ TAXA } & \multicolumn{4}{|c|}{ COLETA } & \multirow[b]{2}{*}{ TAXA } & \multicolumn{4}{|c|}{ COLETA } \\
\hline & 80 & 91 & 92 & 93 & & 80 & 91 & 92 & 93 \\
\hline Família Polyphemidae & & & & & Nauplio & & $\mathrm{X}$ & & \\
\hline Podon polyphemoides & & $x$ & $x$ & $x$ & Ordem Poecilostomatoida & & & & \\
\hline Podon sp. & $x$ & & & & Família Corycaeidae & & & & \\
\hline Evadne tergestina & $x$ & $X$ & $X$ & $x$ & Corycaeus giesbrechti & $X$ & $X$ & $X$ & $x$ \\
\hline E. spinifera & $x$ & $X$ & $X$ & $x$ & Corycaeus sp. & $x$ & $X$ & & \\
\hline E. nordmani & $x$ & & & & Farranula gracilis & & $X$ & $X$ & $x$ \\
\hline Evadne sp. & & $X$ & & & Família Clausidiidae & & & & \\
\hline Classe Copepoda & & & & & Hemicyclops thalassius & & & & $x$ \\
\hline Ordem Calanoida & & $X$ & $X$ & $x$ & Família Oncaeidae & & & & \\
\hline Família Calanidae & & & & $X$ & Oncaea venusta & & $X$ & $X$ & $x$ \\
\hline Família Acartiidae & & & & & Oncaea media & $X$ & $X$ & & \\
\hline Acartia lilljeborgi & $x$ & $x$ & $X$ & $X$ & Oncaea minuta & & $x$ & & \\
\hline Acartia sp. & $x$ & $x$ & & & Oncaea curta & $x$ & & $X$ & $X$ \\
\hline Família Paracalanidae & & & & & Oncaea sp. & $\mathrm{X}$ & $X$ & & \\
\hline Paracalanus quasimodo & $x$ & $X$ & $x$ & $X$ & Ordem Harpacticoida & & & & \\
\hline Paracalanus parvus & $x$ & $x$ & $X$ & $X$ & Família Tachydiidae & & & & \\
\hline Paracalanus crassirostris & $x$ & $\mathrm{X}$ & $x$ & $X$ & Euterpina acutifrons & $\mathrm{X}$ & $\mathrm{X}$ & $X$ & $X$ \\
\hline Paracalanus aculeatus & & $x$ & & $x$ & Macrosetella gracilis & $x$ & & $X$ & $X$ \\
\hline Paracalanus indicus & $x$ & & & & Microsetella norvegica & $x$ & & $x$ & $X$ \\
\hline Acrocalanus longicornis & & $x$ & & & Clytemnestra scutellata & $x$ & & & \\
\hline Família Pseudocalanidae & & & $x$ & & Nauplio & & & $x$ & \\
\hline Clausocalanus furcatus & $x$ & $x$ & $X$ & $X$ & Copepode parasita & & & & $X$ \\
\hline Ctenocalanus citer & & & & $x$ & Classe Cirripedia & $x$ & $x$ & $x$ & $X$ \\
\hline Família Calocalanidae & & & & & Classe Ostracoda & $x$ & $x$ & $X$ & $X$ \\
\hline Calocalanus pavoninus & & $X$ & $X$ & $X$ & Classe Malacostraca & & & & \\
\hline Mecynocera clausi & $x$ & & & & Subclasse Eumalacostraca & & & & \\
\hline Mecynocera sp. & $x$ & & & & Superordem Eucarida & & & & \\
\hline Fam. Pseudodiaptomidae & & & & & Ordem Decapoda & $x$ & $x$ & $X$ & $X$ \\
\hline $\begin{array}{l}\text { Pseudodiaptomus acutus } \\
\text { Família Temoridae }\end{array}$ & $X$ & $x$ & $X$ & $X$ & $\begin{array}{l}\text { Subordem Dendrobranchiata } \\
\text { Infraordem Penaeidea }\end{array}$ & & & & \\
\hline Temora stylifera & $x$ & $x$ & $X$ & $X$ & Família Luciferidae & & $x$ & $X$ & $X$ \\
\hline Família Centropagidae & & & & & Família Sergestidae & $x$ & & & \\
\hline Centropages velificatus & $x$ & $x$ & $X$ & $X$ & Superordem Peracarida & & & & \\
\hline Família Eucalanidae & & & & & Ordem Amphipoda & & & & $X$ \\
\hline Eucalanus pileatus & & $x$ & $x$ & $X$ & Ordem Isopoda & $x$ & & $X$ & $X$ \\
\hline Eucalanus sp. & & & $x$ & $x$ & 3) Filo Chaetognatha & & & & \\
\hline Família Pontellidae & & & & & Sagitta enflata & $x$ & $x$ & $X$ & $X$ \\
\hline Calanopia americana & $x$ & & $x$ & $x$ & Sagitta friderici & $x$ & $x$ & $x$ & $X$ \\
\hline Nauplio & & $x$ & $X$ & $X$ & Sagitta hispida & & $x$ & $X$ & $x$ \\
\hline Ordem Cyclopoida & & & & & Sagitta serratodentata & & $x$ & & \\
\hline Família Oithonidae & & & & & Sagitta hexaptera & $x$ & & & \\
\hline $\begin{array}{l}\text { Oithona hebes } \\
\text { cont. }\end{array}$ & $x$ & $x$ & $X$ & $X$ & $\begin{array}{l}\text { Sagita sp. } \\
\text { Kronitta pacifica }\end{array}$ & $x$ & $\begin{array}{l}X \\
X\end{array}$ & $X$ & $x$ \\
\hline Oithona oculata & $x$ & $x$ & $x$ & $x$ & 4) Filo Cnidaria & & & & \\
\hline Oithona plumifera & $x$ & $x$ & $X$ & $X$ & Classe Hydrozoa & $x$ & $x$ & $x$ & $X$ \\
\hline Oithona sp. & $x$ & $x$ & $x$ & $x$ & Ordem Siphonophora & $x$ & $x$ & $x$ & $X$ \\
\hline
\end{tabular}




\begin{tabular}{|c|c|c|c|c|c|c|c|c|c|}
\hline \multirow[b]{2}{*}{ TAXA } & \multicolumn{4}{|c|}{ COLETA } & \multirow[b]{2}{*}{ TAXA } & \multicolumn{4}{|c|}{ COLETA } \\
\hline & 80 & 91 & 92 & 93 & & 80 & 91 & 92 & 93 \\
\hline 5) Filo Ctenophora & & $\mathrm{X}$ & $\mathrm{X}$ & & Oikopleura longicauda & & $\mathrm{X}$ & $\mathrm{X}$ & $\mathrm{X}$ \\
\hline 6) Filo Mollusca & $\mathrm{X}$ & & & & Oikopleura cophocerca & & $\mathrm{x}$ & $\mathrm{x}$ & \\
\hline Classe Gastropoda & & $x$ & $\mathrm{X}$ & $x$ & Oikopleura sp. & & $\mathrm{x}$ & $\mathrm{x}$ & $\mathrm{X}$ \\
\hline Classe Bivalvia & & $\mathrm{X}$ & $X$ & $\mathrm{X}$ & Subfamília Fritillaridae & $\mathrm{X}$ & & & \\
\hline Classe Pteropoda & $\mathrm{X}$ & $x$ & $\mathrm{X}$ & $x$ & Fritillaria sp. & & $\mathrm{X}$ & $\mathrm{x}$ & $\mathrm{X}$ \\
\hline Classe Heteropoda & & $x$ & & & Classe Thaliacea & & & & \\
\hline Classe Nematoda & & $\mathrm{X}$ & & & Família Salpidae & $x$ & $\mathrm{X}$ & $\mathrm{X}$ & \\
\hline 7) Filo Echinodermata & $\mathrm{X}$ & $\mathrm{X}$ & $\mathrm{X}$ & $\mathrm{X}$ & Família Doliolidae & $x$ & $\mathrm{X}$ & & \\
\hline 8) Filo Chordata & & & & & Doliollum nationalis & & & & $\mathrm{X}$ \\
\hline Subfilo Urochordata & & & & & Classe Ascidiacea & & & $\mathrm{X}$ & $\mathrm{X}$ \\
\hline Classe Larvacea & & & & & Subfilo Cephalochordata & & & & $\mathrm{X}$ \\
\hline Família Oikopleuridae & & & & & Subfilo Vertebrata & & & & \\
\hline Subfamília Oikopleurinae & $\mathrm{x}$ & & & & Superclasse Pisces & & & & \\
\hline Oikopleura dioica & & $X$ & $X$ & $X$ & Classe Osteichthyes & $X$ & $\mathrm{X}$ & $\mathrm{X}$ & $\mathrm{X}$ \\
\hline Oikopleura rufescens & & $\mathrm{X}$ & $\mathrm{X}$ & $\mathrm{X}$ & & & & & \\
\hline
\end{tabular}

As maiores variações da biomassa e densidade de organismos estiveram associadas as variações sazonais de verão e inverno. A análise de grupamento das espécies zooplanctônicas no primeiro ciclo, evidenciou a formação de três grupos sazonais (Fig. 3): A- nos meses de fevereiro a maio (final de verão e outono); Bnos meses de dezembro e janeiro (verão) e; Cnos meses de junho a outubro (inverno $\mathrm{e}$ primavera).

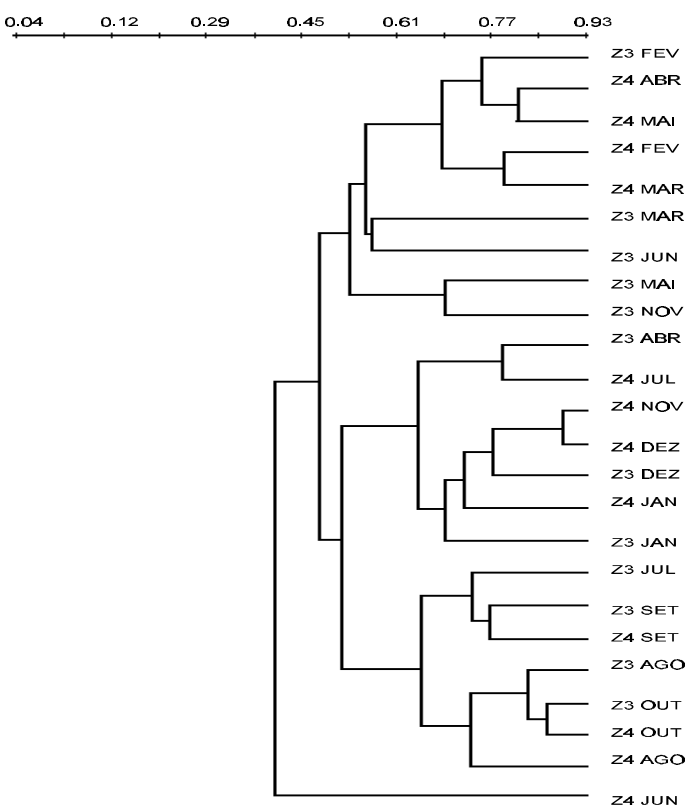

Figura 3: Dendrograma das estações/meses em função dos valores de densidade dos grupos zooplanctônicos do primeiro ciclo de coleta (1980/81).

\section{Segundo ciclo de coleta (1991/93)}

A temperatura variou entre 19 e $28^{\circ} \mathrm{C}$, com máximos nos meses de verão de 1991 e 1992. Em termos gerais, os valores de temperatura registrados na estação Z4 (estação sob a influência das águas usadas na refrigeração do circuito secundário da C.N.A.A.A.) foram sempre superiores, aos encontrados na estação Z3 (estação controle), ao longo de todo o período de coleta (Fig. 4a).

Analisando a variação dos valores de salinidade (Fig. 4b) verificou-se que tendem a seguir o ritmo sazonal das chuvas, variando de 29 a 38 $\%$, tendo os maiores valores de salinidade ocorridos durante o primeiro ano de coleta (1991), com um decréscimo nas demais campanhas. Uma das características marcantes da região é o alto índice pluviométrico, que se revelou maior nos meses de verão e primavera, com índices superiores a $244 \mathrm{~mm}$ de chuva.

O teor de oxigênio dissolvido variou entre 5,18 e $13 \mathrm{mg} . \mathrm{l}^{-1}$ apresentando um decréscimo durante o terceiro ano de coleta (Fig. 4c).

Os valores de clorofila a sofreram grandes oscilações, variando entre 0,37 e $3,29 \mathrm{mg} . .^{-1}$, sendo os maiores valores observados na estação Z3, durante o inverno e a primavera de 1991 (Fig. 4d). 


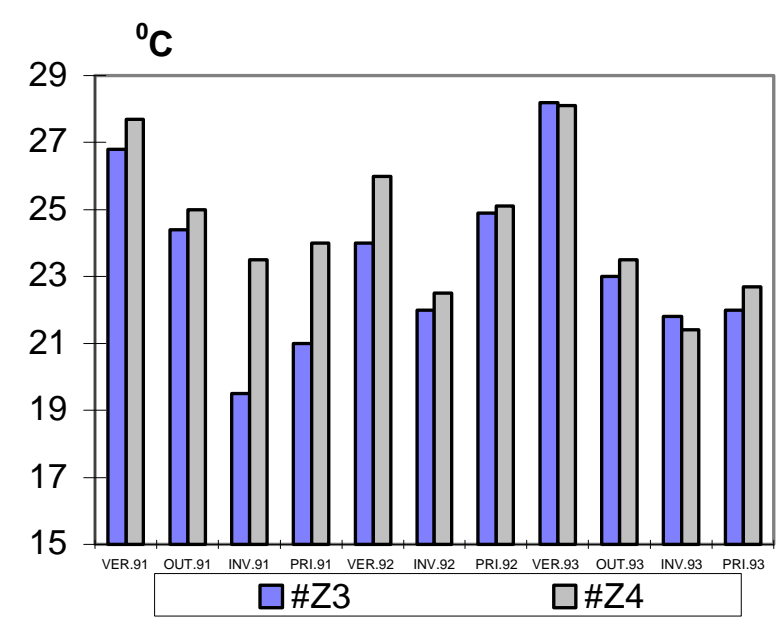

Figura 4a: Variação dos valores de temperatura ao longo do segundo ciclo de coleta (1991/93).

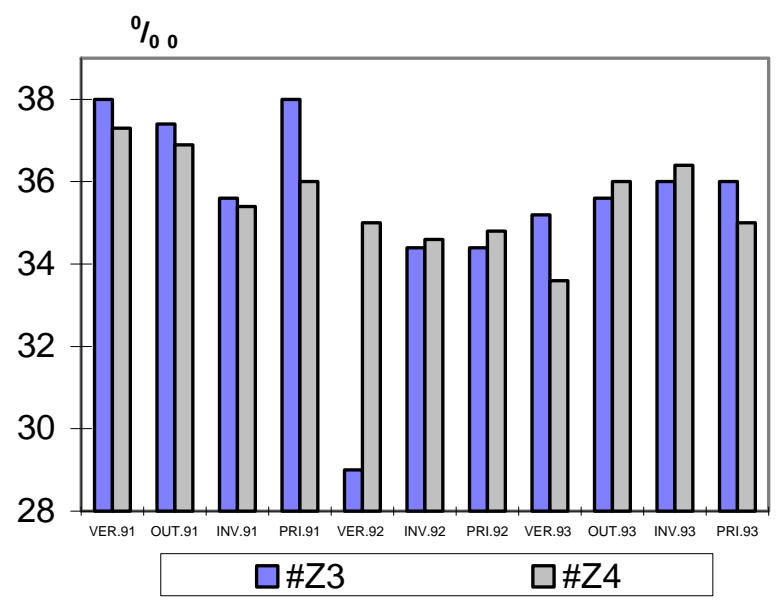

Figura 4b: Variação dos valores de salinidade ao longo do segundo ciclo de coleta (1991/93).

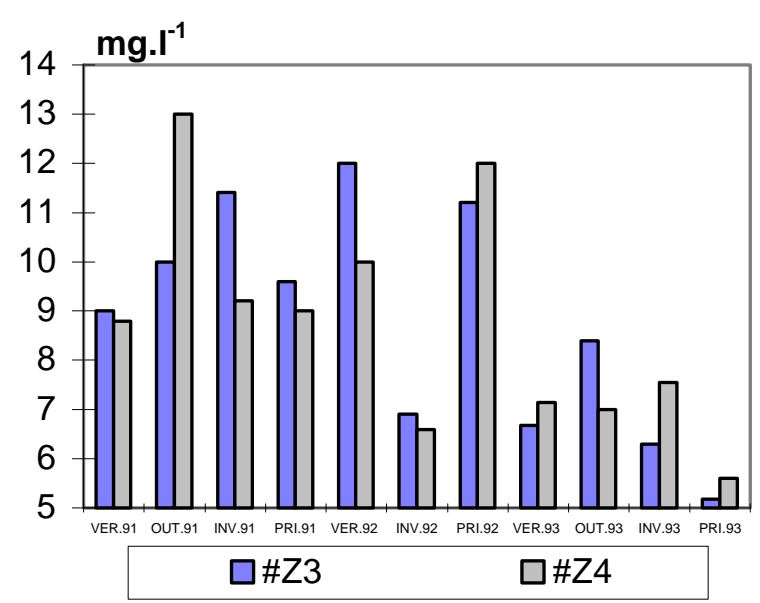

Figura 4c: Variação dos valores de oxigênio dissolvido ao longo do segundo ciclo de coleta (1991/93).

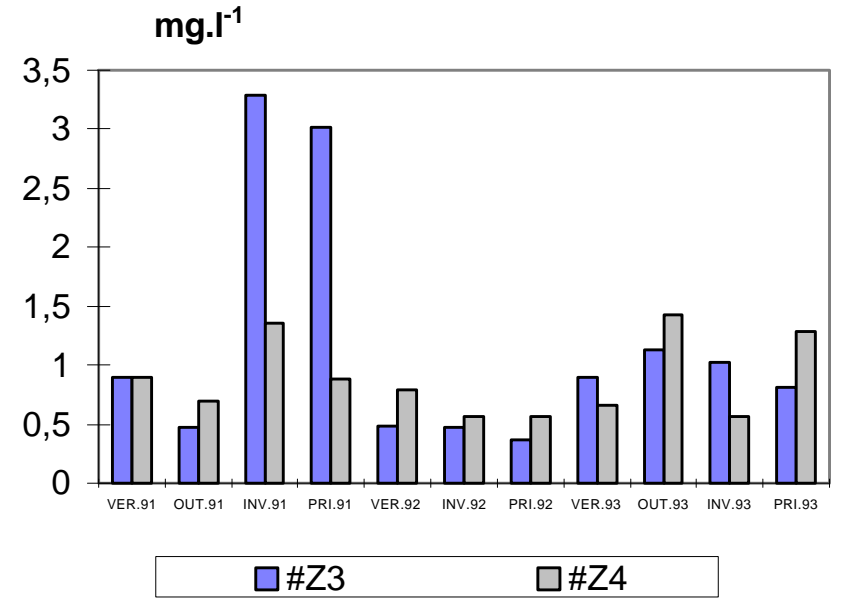

Figura 4d: Variação dos valores de clorofila a ao longo do segundo ciclo de coleta (1991/93).

A densidade do zooplâncton variou de 4.524 a 37.625 ind. $\mathrm{m}^{-3}$, na estação $\mathrm{Z3}$, e de 4.162 a 33.096 ind. . $^{-3}$, na estação Z4 (Fig. 5a e 5b). Os maiores valores foram observados durante as coletas realizadas no verão e na primavera, especialmente durante o segundo ano de coleta. Os Copepoda (47\%) e Cladocera (45\%) constituíram a fração predominante no plâncton.

Os Copepoda dominaram nas épocas de outono e inverno, quando constituíram de 16 a $95 \%$ do zooplâncton total. A densidade variou de $1.428,22$ a $18.639,46$ ind.m ${ }^{-3}$. Um total de 27 espécies de Copepoda foram encontradas durante o estudo, sendo que a grande maioria das espécies foram constituídas por copépodes herbívoros. As espécies pertencentes a família Paracalanidae, Paracalanus parvus copepoditos, $\boldsymbol{P}$. quasimodo e $\boldsymbol{P}$. crassirostris, dominaram em $36 \%$ das amostras. Oithona hebes (27\%), Temora stylifera (23\%), Oncaea curta (9\%) e O. venusta (5\%), foram espécies também importantes no material estudado (Tabela I).

As outras espécies de Copepoda foram frequiêntes, mas com uma baixa densidade. Paracalanus aculeatus, Acrocalanus longicornis, Ctenocalanus citer, Oncaea minuta, $O$. media, Macrosetella gracilis, Microsetella norvegica e Hemicyclops 
thalassius foram consideradas raras, frequiência inferior a $19 \%$.

Os Cladocera foram a fração dominante do zooplâncton, nos meses de primavera e verão, com uma abundância relativa variando de 2 a $73 \%$. Penilia avirostris foi a espécie dominante, com exceção do período de novembro de 1991 a agosto de 1992 quando a espécie Evadne tergestina foi a mais abundante.

As Appendicularia foram o terceiro grupo em importância. O valor máximo de abundância (23\%) foi registrado durante o inverno de 1991. As espécies mais importantes foram Oikopleura dioica, $O$. rufescens e $O$. longicauda.

A)

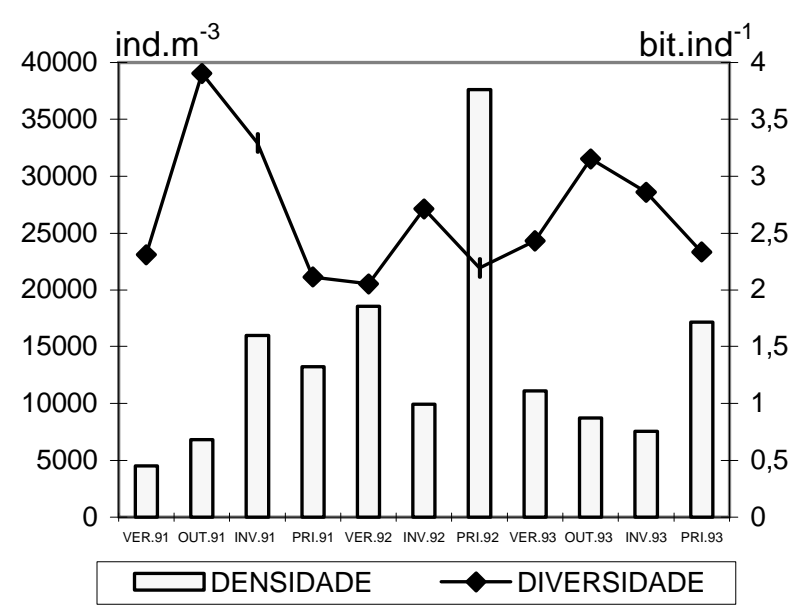

B)

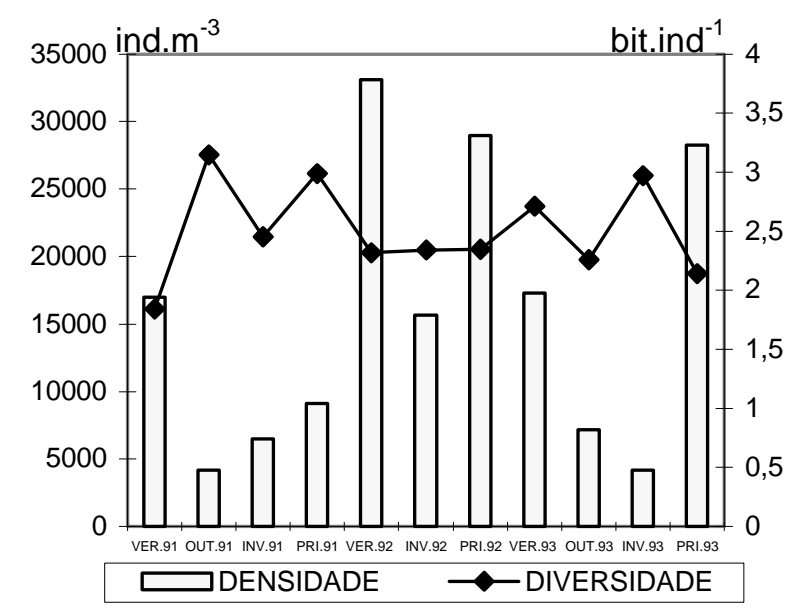

Figura 5: Variação dos valores de densidade e diversidade dos grupos zooplanctônicos ao longo do segundo ciclo de coleta (1991/93): a) estação Z3 e b) estação Z4.

Os Chaetognatha foram um grupo com uma frequiência alta (100\%), embora com um número reduzido de organismos. Sua abundância relativa variou de 0,02 (primavera de 1992) a 1,13\% (verão de 1991). Sagitta friderici, $S$. enflata e $\boldsymbol{S}$. hispida foram as espécies mais abundantes.

O meroplâncton foi muito diversificado. As larvas de Cirripedia (0,07 a 34,36\%) e Mollusca (Gastropoda e Bivalvia) dominaram no material estudado. Larvas de Decapoda, Equinodermata e Polychaeta foram muito freqüentes, porém com abundância inferior. As Larvas de Ascidiacea foram as menos freqüentes e menos abundantes.

Valores extremos de diversidade específica foram observados no primeiro ano de coleta (1991), variando de 1,84 (estação Z3 - verão) a 3,90 bits.ind ${ }^{-1}$ (estação Z4 - outono). Os maiores valores de diversidade específica, superiores a 3,10 bits.ind $^{-1}$, foram registrados durante $\mathrm{o}$ outono e inverno de 1991 e outono de 1993 na estação Z3. A baixa diversidade registrada durante o verão de 1991 foi atribuída a proliferação da espécie $\boldsymbol{P}$. avirostris (Figs. 5a e $5 b)$.

A análise dos valores de densidade para as espécies zooplanctônicas do segundo ciclo de coleta, realizado durante os anos de 1991 a 1993, mostrou a formação de quatro grupamentos (Fig. 6): A- verão de 1991; Boutono de 1991; C- primavera de 1991 e 1993 e verão de 1992 e 1993 e; D- inverno de 1992 e 1993 e primavera de 1992. 


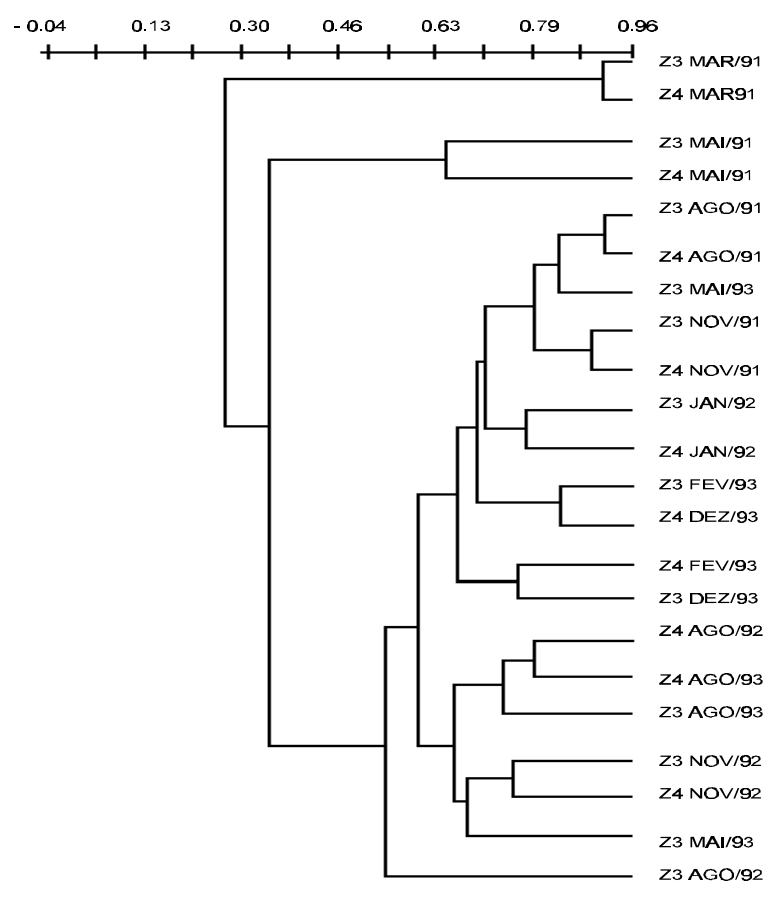

Figura 6: Dendrograma das estações/meses em função dos valores de densidade dos grupos zooplanctônicos do segundo ciclo de coleta (1991/93)

\section{DISCUSSÃO}

A baía da Ribeira é uma região tipicamente tropical, apresentando temperaturas superiores a $19^{\circ} \mathrm{C}$. Segundo Petretski (1987) não existe nenhum curso d'água de porte considerável na região, sendo a formação da água costeira resultante da contribuição da água de origem continental, resultado da lixiviação da costa pelas chuvas, caindo diretamente sobre a massa d'água adjacente.

Comparando os parâmetros analisados com os encontrados durante o ano de 1980 (NOGUEIRA et al., 1987 e 1991), foi observado uma redução da temperatura mínima, de $22^{\circ} \mathrm{C}$ para $19^{\circ} \mathrm{C}$.Com relação ao índice pluviométrico, também foram observadas diferenças. No ciclo 1991/93, foram observados índices aos do ciclo 1980/81. As variações de salinidade acompanharam os resultados sazonais das chuvas da região. Foi observado, também, um aumento de cerca de 5 mg. $l^{-1}$ nos teores de oxigênio dissolvido, em relação ao estudo anterior.
Os valores de densidade do zooplâncton do segundo ciclo de coleta foram 4 vezes maiores aos encontrados no ciclo 1980/81 sem, contudo, ter sido observado alterações na composição zooplanctônica. Este fato pode ser explicado pela utilização, durante o primeiro ciclo de coleta, da Pipeta de Stempel como subamostrador. Este subamostrador não é recomendado para amostragem de organismos grandes (BOLTOVSKOY, 1981), o que poderia ter levado a uma subestimação nos valores de densidade zooplanctônica.

$\mathrm{Na}$ análise dos grupos zooplanctônicos, a presença de organismos holoplanctônicos (Copepoda, Cladocera, Appendicularia, Chaetognatha, entre outros) foi dominante. Os organismos meroplanctônicos tiveram uma alta frequiência e abundância, sendo esta uma das características das populações zooplanctônicas de sistemas estuarinos (TUNDISI, 1970). Dos grupos zooplanctônicos encontrados, os Copepoda constituíram a fração dominante nos dois ciclos de coleta, sendo este fato esperado, uma vez que a distribuição dos organismos deste grupo em regiões costeiras pode constituir de 60 a $90 \%$ do total do zooplâncton (JEFFRIES, 1967).

O zooplâncton apresentou um padrão de sazonalidade definido. Os Copepoda mostraram máximos de densidade no outono e inverno, sendo que no verão e primavera os percentuais de abundância relativa diminuíram, com os Cladoceros passando a serem os organismos mais representativos. Em relação aos Copepoda pode-se observar um aumento no número de espécies encontradas do primeiro para o segundo ciclo de coleta, sem haver, entretanto, uma grande variação entre as espécies mais representativas nos dois ciclos de coleta.

Os grupos zooplanctônicos foram representados por organismos preferencialmente herbívoros e por formas filtradoras. No segundo ciclo de coleta (1991/93), os maiores valores para a clorofila a foram registrados nos meses de maio e agosto, meses estes que apresentaram as maiores abundâncias relativas para os Copepoda. Segundo Razouls (1975) as variações de Copepoda ocorrem em função do ciclo de 
fitoplâncton e da dinâmica de diversas espécies na comunidade planctônica.

Durante o segundo ciclo de coleta foi encontrado um maior número de grupos zooplanctônicos do que os coletados durante o ano de 1980. O ictioplâncton foi melhor representado do que no período anterior, quando não foram coletados nos meses de outubro, abril, maio e julho (FUJB, 1981). Apesar de sua alta frequiência, os ovos e larvas de peixe apresentaram uma baixa abundância relativa, nos dois ciclos de coleta, que pode ser explicada pela forma de arrasto utilizado, arrasto vertical, não ser apropriado para a coleta deste tipo de material (PHONLOR, 1973).

Tendo em vista que Nogueira et al. (1991) calcularam a diversidade específica, para o primeiro ciclo de coleta, utilizando apenas as espécies de Copepoda, o mesmo foi recalculado, agora utilizando-se as espécies de Copepoda, Cladocera e Chaetognatha. Como o índice calculado foi bastante semelhante aquele determinado por Nogueira et al. (1991), optou-se por utilizá-lo para uma análise comparativa com o utilizado para o segundo ciclo de coleta, calculado, também, a partir das espécies dos três grupos acima citados. Assim sendo, observou-se que a diversidade específica, no período de 1991/93, apresentou índices superiores aos encontrados no primeiro ciclo de coleta. Nos dois ciclos de coleta os maiores valores de diversidade foram observados, principalmente, nos meses frios.

As usinas termo-elétricas podem afetar os ecossistemas de várias maneiras, sendo que a principal forma seria devido ao aumento da temperatura da água, em estuários ou baías, onde estariam situados os pontos de descarga dos seus efluentes (BARNETTE, 1972). O aumento da temperatura selecionaria os organismos mais tolerantes as suas variações, o que acarretaria a diminuição da densidade geral (FOX \& MOYER, 1973).

Outra forma de impacto é a passagem do zooplâncton através dos condensadores, que poderia causar danos a estrutura desses organismos (YOUNGBLUTH, 1976).
No presente trabalho não foram observadas variações na comunidade zooplanctônica, em relação a atividade da Usina Angra I.

Alguns estudos como os de Paranaguá et al. (1979) no Estuário do Rio Igarassu; Paranaguá e Nascimento-Vieira (1984) no Estuário do Rio Botafogo e Nogueira et al. (1989) na Baía de Guanabara, apresentaram associações planctônicas semelhantes as encontradas no presente estudo.

A evolução do zooplâncton na região mostrou uma variação sazonal, ligada ao período de crescimento populacional, com predomínio de organismos holoplanctônicos (Copepoda e Cladocera), sem haver um processo de competição entre esses organismos. Comparando os grupamentos encontrados na análise do primeiro ciclo de coleta, realizado com valores de densidade dos grupos zooplanctônicos Copepoda, Cladocera e Chaetognatha, com os de Nogueira et al. (1991), que utilizou os valores de densidade de Copepoda, deste mesmo período, pode-se observar uma coincidência dos mesmos, o que demonstra a representatividade do grupo selecionado para a análise (Copepoda). Em relação ao segundo ciclo de coleta, nota-se uma maior individualização dos grupamentos formados pelos meses quentes. Esta sazonalidade estaria ligada, principalmente, ao regime das chuvas que caem na região, que seria o fator que afetaria a abundância das populações do zooplâncton.

\section{AGRADECIMENTOS:}

A FURNAS Centrais Elétricas S/A pela coleta do material zooplanctônico e pelo fornecimento dos dados físicos, químicos, biológicos e pluviométricos. Aos laboratórios de zooplâncton dos Departamentos de Zoologia e Biologia Marinha (Instituto de Biologia-UFRJ) pelo apoio no desenvolvimento deste trabalho. Ao biólogo Luis Roberto Silveira Cunha Vasconcelos pela identificação do grupo Chaetognatha. 


\section{REFERÊNCIAS BIBLIOGRÁFICAS}

Barnette, P.R.O. (1972), Effects of warm water effluents from power stations on marine life. Proc. R. Soc. (Ser. B), 180: 497-509.

Boltovskoy, D. (1981), Submuestreo. In: Boltovskoy. Atlas del Zooplancton del Atlantico Sudoccidental y metodos de trabajo com el zooplancton marino. INIDEP, Mar del Plata, Argentina, p: 143-146.

Fox, J.L.; Moyer, M.S. (1973), Some Effects of a Power Plant on Marine Microbiota. Chesapeake Sci. 14(1): 1-10.

FUJB. (1981), Análise Biológica na Área da CNAAA - Unidade I. Relatório Conclusivo de Plâncton (1980-1981). Fundação Universitária José Bonifácio/UFRJ. 165 pp.

Jeffries, H. P. (1967), Saturation of estuarine zooplankton by congeneric associates. In: G. H. Lauff (ed.) Estuaries, Washington, p: 500-508.

Legendre, L.; Legendre, P. (1983), Numerical ecology. Elsevier. Scient. Publ. Company, New York, $419 \mathrm{pp}$.

Nogueira, C. R.; Bonecker, A. C. T.; Bonecker, S. L. C.; Silva, V. M. A. P. da; Skinner-Neto, G. (1987), Zooplâncton da Baía da Ribeira. Avulso do Depto. Zool. UFRJ-IB-UFRJ, 34: 1-20.

Nogueira, C. R.; Bonecker, A. C. T.; Bonecker, S. L. C. (1989), Zooplâncton da Baía de Guanabara (RJ - Brasil) - Composição e variações espaçotemporais. In: F. P. Brandini (ed.) Memorias do III EBP, Curitiba, p: 151-156.

Nogueira, C. R.; Bonecker, A. C. T.; Bonecker, S. L. C.; Santos, C.C. (1991), Studies of Zooplankton near the Nuclear Power Plant - Angra I. Preoperational Conditions (Rio de Janeiro, Brazil). In: Magoon, O. (ed.). Coastal Zone'91 Publ. American Society of Civil Engineers, Long Beach, vol. 4: 3221-3233.

Paranaguá, M. N.; Nascimento, D. A. do; Macêdo, S. J. de 91979), Estudo ecológico da região de
Itamaracá, Pernambuco, Brasil. II. Distribuição do zooplâncton no estuário do Rio Igarassu. Trab. Oceanogr. Univ. Fed. PE., Recife, 14: 65-92.

Paranaguá, M. N.; Nascimento-Vieira, D. A. DO (1984), Estudo ecológico da região de Itamaracá, Pernambuco-Brasil. XXV. Zooplâncton do Rio Botafogo. Trab. Oceanogr. Univ. Fed. PE., Recife, 18: 193-206.

Petretski, J. H. (1987), Contribuição ao estudo das comunidades fitoplanctônicas da região a ser afetada pela Central Nuclear Almirante Álvaro Alberto, Angra dos Reis, Rio de Janeiro. Verificação do grau de homegeinidade da distribuição fitoplanctônica da Enseada de Piraquara de Fora (Angra dos Reis, RJ). Monografia para obtenção do grau de Bacharel em Biologia Marinha pelo Depto de Biologia Marinha do Instituto de Biologia da Universidade Federal do Rio de Janeiro. p: 1-34.

Phonlor, G. (1973), Estudo preliminar do ictioplâncton. Publ. esp. Inst. Oceanogr. S.Paulo. 3(1): 429-464.

Razouls, C. (1975), Estimation de la prodution globale de Copépodes planctoniques dans la province néritique du Golfe du Lion Banyuls - sur - Mer. II Variations annuelles de la biomasse et calcul de la production. Vie et Milieu, ser. B: Océanographie, Paris, 25(1): 99-122.

Tundisi, J. (1970), O plâncton estuarino. Contr. Inst. Oceanogr. Univ. S. Paulo ser. Oceanogr. Biol., 19: 1-12.

Youngbluth, M.J. (1976), Zooplankton Population in a Polluted Tropical Embayment. Estuar. Coast. Mar. Sci., 4: 481-496. 